\title{
Comparison of treatment outcomes in lumbar central stenosis patients treated with epidural steroid injections: interlaminar versus bilateral transforaminal approach
}

\author{
Savas Sencan ${ }^{1}$, Ipek Saadet Edipoglu ${ }^{1}$, Alp Eren Celenlioglu ${ }^{2}$, Gunay Yolcu ${ }^{3}$, and Osman Hakan Gunduz ${ }^{1}$ \\ 'Division of Pain Medicine, Department of Physical Medicine and Rehabilitation, Faculty of Medicine, Marmara University, Istanbul, Turkey \\ ${ }^{2}$ Division of Pain Medicine, Department of Physical Medicine and Rehabilitation, Faculty of Medicine, Erciyes University, Kayseri, Turkey \\ ${ }^{3}$ Department of Physical Medicine and Rehabilitation, Faculty of Medicine, Marmara University, Istanbul, Turkey
}

Received November 28, 2019

Revised March 8, 2020

Accepted March 10, 2020

Handling Editor: Won Hyung Lee

\section{Correspondence}

Ipek Saadet Edipoglu

Division of Pain Medicine, Department of Physical Medicine and Rehabilitation, Marmara University Pendik Education and Research Hospital, Muhsin

Yazıcıoğlu Cad. No: 10, Üst Kaynarca, 34899 Pendik/Istanbul, Turkey

Tel: +90-216-625-4545

Fax: +90-216-414-4731

E-mail: dripeks@yahoo.com
Background: We aimed to compare interlaminar epidural steroid injections (ILESI) and bilateral transforaminal epidural steroid injections (TFESI) on pain intensity, functional status, depression, walking distance, and the neuropathic component in patients with lumbar central spinal stenosis (LCSS).

Methods: The patients were divided into either the ILESI or the bilateral TFESI groups. Prime outcome measures include the numerical rating scale (NRS), Oswestry disability index (ODI), Beck depression inventory (BDI), and pain-free walking distance. The douleur neuropathique en 4 questions score was used as a secondary outcome measure.

Results: A total of 72 patients were finally included. NRS, ODI, and BDI scores showed a significant decline in both groups in all follow-ups. Third-month NRS scores were significantly lower in the ILESI group $(P=0.047)$. The percentages of decrease in the ODI and BDI scores between the baseline and the third week and third month were significantly higher in the ILESI group $(P=0.017, P=0.001$ and $P=0.048, P=0.030$, respectively). Pain-free walking distance percentages from the baseline to the third week and third month were significantly higher in the ILESI group $(P=0.036, P<0.001)$. The proportion of patients with neuropathic pain in the bilateral TFESI group significantly decreased in the third week compared to the baseline $(P=0.020)$.

Conclusions: Both ILESI and TFESI are reliable treatment options for LCSS. ILESI might be preferred because of easier application and more effectiveness. However, TFESI might be a better option in patients with more prominent neuropathic pain.

Key Words: Chronic Pain; Depression; Injections, Epidural; Low Back Pain; Lumbosacral Region; Neuralgia; Pain Management; Patient Outcome Assessment; Spinal Stenosis.

\section{INTRODUCTION}

Lumbar central spinal stenosis (LCSS) is the narrowing of the spinal canal with encroachment on the neural struc- tures by surrounding bone and soft tissues. It is a multifactorial disorder prevalent in $27.2 \%$ of the population [1]. Clinical presentation can be variable with or without neurogenic claudication (NC), manifested by pain in the but- (a) This is an open-access article distributed under the terms of the Creative Commons Attribution Non-Commercial License (http://creativecommons.org/licenses/by-nc/4.0/), which permits unrestricted non-commercial use, distribution, and reproduction in any medium, provided the original work is properly cited.

(C) The Korean Pain Society, 2020
Author contributions: Savas Sencan: Writing/manuscript preparation; Ipek Saadet Edipoglu: Writing/manuscript preparation; Alp Eren Celenlioglu: Writing/manuscript preparation; Gunay Yolcu: Data curation; Osman Hakan Gunduz: Supervision. 
tocks or legs when walking, which disappears with sitting or lumbar flexion [2]. Regardless of the etiology, LCSS can cause chronic pain and disability, as well as a dramatically reduced quality of life, mobility, and function [3].

Epidural steroid injections (ESI) are administered for pain management after other conservative modalities, including analgesic medications, exercise, physical therapy, and/or chiropractic adjustments, have failed. They are performed by three different approaches, namely the caudal, lumbar interlaminar, and lumbosacral transforaminal. Assessments of interlaminar ESI (ILESI) and transforaminal ESI (TFESI) have shown variable results in managing LCSS. Lee et al. [4] reported that bilateral TFESI is a more effective treatment method than ILESI in patients with spinal stenosis. In contrast, Smith et al. [5] reported that neither TFESI nor ILESI resulted in superior short-term pain improvement, or fewer long-term surgical interventions or repeated injections when compared with each other.

Chronic low back pain is characterized by a combination of neuropathic and nociceptive pain mechanisms [6]. People with neuropathic pain (NP) had higher levels of functional impairment and psychopathology [7]. Therefore, it is important to identify effective treatment strategies for NP. Rados et al. [8] compared the efficacy of the two ESI methods for chronic radicular pain using the painDETECT questionnaire. They suggested that steroids are efficient; besides alleviating the overall pain, they also reduce the neuropathic component of chronic lumbar radicular pain, whether it is distributed epidurally by the interlaminar or transforaminal approach. To the best of our knowledge, there are no data investigating the effects of transforaminal and interlaminar ESI on the NP component in patients with LCSS.

In this study, our primary aim was to compare the effects of bilateral TFESI and ILESI on pain and walking distance, as well as functional and depression status in patients with NC due to LCSS. Our secondary aim was to compare the efficacy of bilateral TFESI and ILESI on the NP component of pain in these patients.

\section{MATERIALS AND METHODS}

This study was conducted in the Division of Pain Medicine, Department of Physical Medicine and Rehabilitation in the Marmara University Pendik Education and Research Hospital between September 2018 and April 2019. The study was approved by the Ethics Committee of Marmara University (No.: 09.2018.590; approval date: January $13,2019)$. All patients were informed about the study and their verbal and written consent were obtained.
Study participants were recruited from consecutive patients between the ages of 40 and 75 years with a diagnosis of multilevel degenerative LCSS and a history of chronic $\mathrm{NC}$, including bilateral buttocks and/or bilateral lower extremity aching/heaviness/tiredness in a diffuse distribution, with or without low back pain during prolonged standing or walking, and relieved by leaning forward or sitting. The multilevel degenerative LCSS clinical diagnosis was confirmed by evidence on magnetic resonance imaging (MRI) at least moderate and two-level central canal stenosis as documented by neuroradiologists who had no knowledge of this study. A simple and frequently used method for quantifying central stenosis is the crosssection area of the dural sac. When this area is $100 \mathrm{~mm}^{2}$, the stenosis is considered moderate; when it is $75 \mathrm{~mm}^{2}$, the stenosis is considered severe [9]. The patients were randomized into two groups with a randomization software package. The first group underwent bilateral TFESI and the second group underwent ILESI. The level below the most stenotic level was chosen, because we did not want to create increased pressure by injecting at the most stenotic level, and we found that contrast medium spread to the epidural area is more cephalad than caudad.

Exclusion criteria were as follows: (1) Radiologic spinal stenosis without NC; (2) patients with unilateral lancinating pain in the pattern of a single nerve root distribution; (3) vascular claudication; (4) history of previous lumbosacral surgery; (5) history of polyneuropathy or entrapment neuropathy; (6) presence of systemic or local infection, systemic diseases such as ankylosing spondylitis, rheumatoid arthritis or diabetes mellitus; (7) receipt of lumbar ESI within the past 6 months; (8) dynamic/postural radiculopathy attributable to foraminal and/or lateral recess stenosis.

\section{Assessment scales}

Demographic and clinical characteristics including age, sex, body mass index, symptom duration, most painful side, most stenotic level, stenosis degree, and medication were recorded for all patients. Besides, the pain intensity was evaluated using the numerical rating scale (NRS) before the intervention and in the first hour, third week, and third month after the intervention. The functional characteristics were evaluated with the Oswestry disability index (ODI) before the intervention and in the third week and third month following intervention. At the same follow-up examinations, depressive mood assessment was done with the Beck depression inventory (BDI). In addition, patients were permitted to walk on the walking track before the intervention and in the third week and third month after the intervention, and the distance they could walk before the 
emergence of pain related to NC was recorded. The presence of NP was evaluated with douleur neuropathique en 4 questions (DN4) before the intervention and at the third week and third month after the intervention. A DN4 score equal to or greater than 4 was accepted as indicating the presence of NP. The number of patients who had NP according to the DN4 score has been considered.

The post-intervention follow-up assessments were done by a physiatrist, who was blind to the initial examinations and performed procedures. Patients were not informed about the injection type they were receiving in order to make them blind to the treatments. The injections were made by a pain specialist with 10 years of experience under fluoroscopy. Throughout the study, patients did not receive any medication for nociceptive or NP and the existing drugs of patients were not changed.

\section{Interventions}

All patients were placed in the prone position and supported with a pillow under the abdomen to reduce lumbar lordosis. The injection site was cleaned thrice with povidone iodine $10 \%$ solution, and covered with sterile drapes. For TFESI, the fluoroscopy device was positioned with a 10-30 degree oblique and 0-15 degree craniocaudal angle, and the neural foramen was visualized. The injection site and subcutaneous tissue were infiltrated with a local anesthetic ( $3 \mathrm{~mL} 2 \%$ prilocaine). A $22 \mathrm{G} 8.89 \mathrm{~cm}$ spinal needle was inserted to the subpedicular area under intermittent fluoroscopic visualization with the co-axial technique (directed to the 6 o'clock position). While approaching the epidural area, the position of the needle was confirmed with lateral imaging. For ILESI, the optimal visualization of the interlaminar space was obtained by adjusting the cranio-caudal angle of the fluoroscopic beam to align the endplates of the targeted vertebrae. Afterwards, the epidural space was accessed via the parasagittal approach, using the loss-of-resistance technique.

During both interventions, 1 to $2 \mathrm{~mL}$ contrast dye was given to confirm that the needle was in the epidural space in the posteroanterior and lateral images. After the confirmation of the presence of the epidural distribution without vascular distribution, a mixture of $80 \mathrm{mg}$ methylprednisolone acetate, $2 \mathrm{~mL}$ saline solution, and $2 \mathrm{~mL}(0.5 \%)$ bupivacaine solution was injected (ILESI). For TFESI, the same mixture was divided into two equal doses and injected into the right and left foramina at the related level. After the intervention, patients were referred to the observation room and followed for one hour. Patients, in whom no complications developed, were discharged following their first-hour examination with recommendations.

\section{Statistical analysis}

The descriptive data were expressed in numbers and percentages, and continuous data were expressed in median interquartile range (25th-75th percentiles). A chi-square test was used to compare categorical data. The obtained data were tested for the normal distribution with the Kolmogorov-Smirnov test. The non-normal distributed data were compared with the Mann-Whitney $U$-test and Friedman's test where appropriate. For all analyses, $P<$ 0.05 was considered statistically significant. The analyses were done with SPSS ver. 21.0 (IBM Corp., Armonk, NY). Power and Sample Size Program (PS ver. 3.1.2) were used for the analysis of the patient number. As the change in the NRS scale was predicted as $50 \%$, a minimum of 62 patients were needed for an $\alpha=0.05$ and power $=0.80$ in the final analysis. For this patient number analysis, we referred to the study conducted by Manchikanti et al. [10].

\section{RESULTS}

Seventy-two patients were included in the study. Two patients in the ILESI group and 3 patients in the bilateral TFESI group were excluded from the study, as they did not follow the control schedule after the injection. A total of 67 patients (ILESI group $=34$ [50.7\%], the bilateral TFESI group $=33[49.3 \%])$ completed the study. The median age was 63 and 62 years in the ILESI and TFESI groups, respectively. The median duration of symptoms was 29 and 24 months in the ILESI and bilateral TFESI groups, respectively. There was no statistically significant difference between the groups for the demographic and clinical characteristics (Table 1). The MRI examination showed that the most stenotic level was between L3 and L4 in 23 patients (ILESI: 13; TFESI: 10), and between L4 and L5 in 44 patients (ILESI: 21; TFESI: 23). Both groups were similar according to the degree of stenosis (Table 1 ).

The evaluation of NRS scores showed that a significant decline was achieved in both groups in all follow-up examinations $(P<0.001)$. The third-month scores were significantly lower in the ILESI group compared to the bilateral TFESI group $(P=0.047)$. Besides, the percentage of the decrease in the pain score between the baseline and the third month was significantly higher in the ILESI group than the bilateral TFESI group $(P=0.019$, Table 2$)$.

The evaluation of the intergroup changes of ODI and BDI scores revealed that there was a significant decline in the scores of both groups in all follow-up examinations ( $P$ $<0.001)$. Furthermore, the percentages of decrease in the ODI and BDI scores between the baseline and the third week and third month were significantly higher in the 
Table 1. The Evaluation of the Demographic and Clinical Characteristics in the Study Groups

\begin{tabular}{|c|c|c|c|c|}
\hline \multicolumn{2}{|c|}{ Characteristic } & \multirow{2}{*}{$\begin{array}{r}\text { ILESI } \\
8(23.5)\end{array}$} & \multirow{2}{*}{$\begin{array}{c}\text { TFESI } \\
10(30.3)\end{array}$} & \multirow{2}{*}{$\begin{array}{l}P \text { value } \\
0.532\end{array}$} \\
\hline Sex & $M$ & & & \\
\hline & $\mathrm{F}$ & $26(76.5)$ & $23(69.7)$ & \\
\hline Age (yr) & & $63.0(62.0-65.0)$ & $62.0(54.0-65.0)$ & 0.080 \\
\hline $\operatorname{BMI}\left(\mathrm{kg} / \mathrm{m}^{2}\right)$ & & $24.6(23.0-31.2)$ & $26.1(24.7-29.8)$ & 0.404 \\
\hline Symptom duration ( & & $29.0(24.0-36.0)$ & $24.0(12.0-36.0)$ & 0.664 \\
\hline Most painful side ( $r$ & & $14 / 9^{a}$ & $12 / 10^{\mathrm{a}}$ & \\
\hline \multirow[t]{2}{*}{ Most stenotic level } & L3-L4 & $13(38.2)$ & $10(30.3)$ & 0.125 \\
\hline & L4-L5 & $21(61.8)$ & $23(69.7)$ & 0.241 \\
\hline \multirow[t]{2}{*}{ Stenosis degree } & Moderate & $21(61.8)$ & $22(66.7)$ & 0.487 \\
\hline & Severe & $13(38.2)$ & $11(33.3)$ & 0.125 \\
\hline \multirow[t]{3}{*}{ Medication $^{\mathrm{b}}$} & NSAID & $12(35.3)$ & $10(30.3)$ & 0.307 \\
\hline & Paracetamol & $14(41.2)$ & $15(45.5)$ & 0.310 \\
\hline & Pregabalin/gabapentin & $16(47.1)$ & $17(51.5)$ & 0.499 \\
\hline
\end{tabular}

Values are presented as number (\%), median (range), or number only.

Categorical data were analyzed by the chi-square test, and continuous data were analyzed by the Mann-Whitney U-test.

ILESI: interlaminar epidural steroid injection, TFESI: transforaminal epidural steroid injection, BMI: body mass index, NSAID: non-steroid anti-inflammatory drug.

${ }^{a}$ The other patients have middle or bilateral side pain. ${ }^{b}$ The sum of the percentages does not equal $100 \%$ because of multiple responses.

Table 2. Comparison of the Changes in the NRS, ODI, BDI, and Walking Distance in the Study Groups

\begin{tabular}{|c|c|c|c|c|c|c|c|c|}
\hline \multirow{3}{*}{ Clinical parameter } & \multicolumn{6}{|c|}{ Group } & \multirow{3}{*}{$P$ value ${ }^{a}$} & \multirow{3}{*}{$P$ value $^{\mathrm{b}}$} \\
\hline & \multicolumn{3}{|c|}{ ILESI } & \multicolumn{3}{|c|}{ TFESI } & & \\
\hline & Median & Range & $\%$ & Median & Range & $\%$ & & \\
\hline \multicolumn{9}{|l|}{ NRS } \\
\hline Baseline & 9.0 & 8.0-10.0 & - & 9.0 & 8.0-10.0 & - & 0.906 & - \\
\hline After the injection & 2.0 & $0.0-3.0$ & 80.0 & 0.0 & $0.0-2.0$ & 100 & 0.114 & 0.135 \\
\hline Third wk & 3.0 & $0.0-5.0$ & 66.7 & 4.0 & $0.0-5.0$ & 50.0 & 0.278 & 0.145 \\
\hline Third mo & 4.0 & $1.0-5.0$ & 55.6 & 5.0 & $3.5-9.0$ & 50.0 & 0.047 & 0.019 \\
\hline$P$ value $^{c}$ & $<0.001$ & & & $<0.001$ & & & & \\
\hline \multicolumn{9}{|l|}{ ODI } \\
\hline Baseline & 26.5 & $18.0-34.0$ & - & 24.0 & $14.0-29.0$ & - & 0.309 & - \\
\hline Third wk & 18.0 & $12.0-23.0$ & 36.2 & 19.0 & $12.0-23.0$ & 20.8 & 0.925 & 0.017 \\
\hline Third mo & 18.5 & $11.0-26.0$ & 34.8 & 19.5 & $12.0-27.5$ & 9.6 & 0.649 & 0.001 \\
\hline$P$ value $^{c}$ & $<0.001$ & & & $<0.001$ & & & & \\
\hline \multicolumn{9}{|l|}{$\mathrm{BDI}$} \\
\hline Baseline & 16.5 & $12.0-23.0$ & - & 19.0 & $15.0-27.0$ & - & 0.101 & - \\
\hline Third wk & 12.0 & 8.0-17.0 & 21.8 & 17.0 & $12.0-19.0$ & 13.0 & 0.048 & 0.064 \\
\hline Third mo & 13.0 & $7.0-19.0$ & 21.4 & 17.5 & $11.0-23.0$ & 12.4 & 0.030 & 0.138 \\
\hline$P$ value $^{c}$ & $<0.001$ & & & $<0.001$ & & & & \\
\hline \multicolumn{9}{|l|}{ WD (m) } \\
\hline Baseline & 75 & $50-100$ & - & 100 & $50-100$ & - & 0.640 & - \\
\hline Third wk & 175 & $100-300$ & 100 & 150 & $100-200$ & 50 & 0.232 & 0.036 \\
\hline Third mo & 150 & $75-250$ & 100 & 100 & $50-150$ & 0 & 0.048 & $<0.001$ \\
\hline$P$ value $^{c}$ & $<0.001$ & & & $<0.001$ & & & & \\
\hline
\end{tabular}

ILESI: interlaminar epidural steroid injection, TFESI: transforaminal epidural steroid injection, NRS: numerical rating scale, ODI: Oswestry disability index, BDI: Beck depression inventory, WD: walking distance, -: not available.

${ }^{\mathrm{a}}$ Mann-Whitney U-test (for comparison of median values between groups). ${ }^{\mathrm{b}}$ Mann-Whitney U-test (for comparison of median percentage of changes between groups). 'Friedman's test (for comparison of intragroup median values during follow-up periods).

ILESI group compared to the bilateral TFESI group $(P=$ $0.017, P=0.001$ and $P=0.048, P=0.030$, respectively; Table 2).

The walking distance increased significantly in both groups in the third week $(P<0.001)$. The intergroup comparison showed that the distance in the third month was significantly longer in the ILESI group $(P=0.048)$. Besides, the percentage of the increase in the walking distance from the baseline to the third week and third month were significantly higher in the ILESI group $(P=0.036, P<0.001)$ (Table 2).

NP was encountered in $58.2 \%$ of total patients, $69.7 \%$ in 


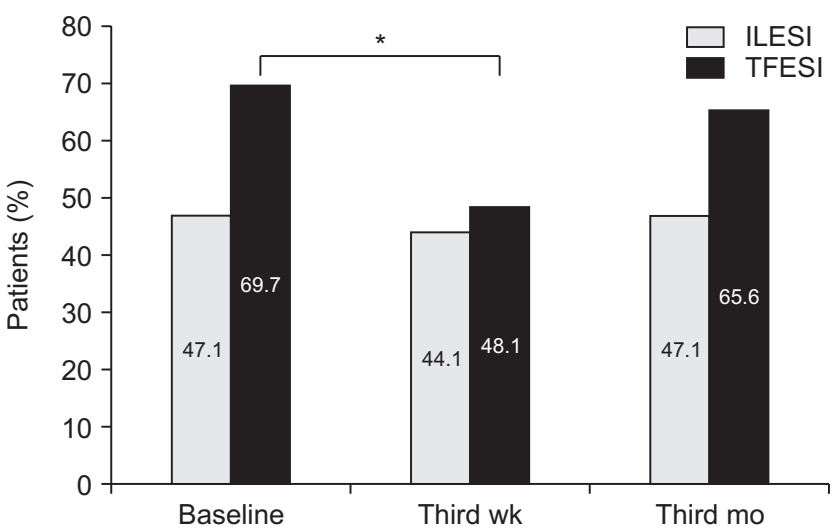

Fig. 1. Percentage of patients showing NP (DN4 score $>4$ ) at different time intervals in ILESI and TFESI groups. Cochran $\mathrm{Q}$ and chi-square test were used for comprasion of in groups and between groups, respectively. NP: neuropathic pain, DN4: douleur neuropathique en 4 questions, ILESI: interlaminar epidural steroid injection, TFESI: transforaminal epidural steroid injection. *Patients with neuropathic pain were significantly reduced from baseline in TFESI group at week $3(P<0.05)$.

the bilateral TFESI group, and $47.1 \%$ in the ILESI group. However, there was no statistically significant difference between the groups. While no significant change occurred in the number of patients with NP in the ILESI group, the proportion of patients with NP decreased significantly in the bilateral TFESI group in the third week compared to the baseline ( $P=0.020$, Fig. 1 ).

After the intervention, hypotension due to vasovagal reactions emerged in 2 patients in the bilateral TFESI group and one patient complained of pain at the injection site in the ILESI group. Additionally, lower extremity motor blocks were seen in 3 patients in the bilateral TFESI group due to selective nerve blockage. After the short-term follow-up, all patients, whose symptoms were relieved and findings improved, were discharged with recommendations.

\section{DISCUSSION}

In our study, we compared the effects of bilateral TFESI and ILESI on the walking distance, functional status, and depression, along with pain intensity and the neuropathic component of pain mechanisms in patients with NC due to LCSS. In both injection groups, a significant decrease in pain intensity, BDI and ODI scores, and a significant increase in the walking distance were observed during all follow-up periods. Furthermore, the decrease in pain intensity, ODI and BDI scores and the increase in the walking distance were significantly greater in the third month compared to the baseline in the ILESI group in contrast to the bilateral TFESI group. While $69.7 \%$ of patients, who un- derwent bilateral TFESI, had NP before the treatment, the same rate was $47.1 \%$ in the ILESI group. The number of patients, whose NP was relieved after the injection decreased only in the bilateral TFESI group in the third week.

TFESI provide important advantages, like a higher amount of distribution in the anterior epidural area, and injection of a maximum amount of the injectate directly to the targeted area [11]. Therefore, many authors are recommending the TFESI method for ESI in patients with CSS $[12,13]$. Farooque et al. [12] published the results of a 6-month follow-up of 22 patients, who had lumbar degenerative CSS and were treated with bilateral TFESI and reported that this approach provided a moderate improvement in pain and functional status. In our study, although patients were followed for a shorter period, as in the above-mentioned study, we observed a decrease in pain, improvement in functional status and depression in all follow-up periods in the bilateral TFESI group along with improvement in the walking distance, which had been decreased due to the NC. Bilateral TFESI is recommended in patients with LCSS, who have NC and axial pain, as the injectate accumulates in this area due to the barrier formed by the meningovertebral ligaments [14]. Therefore, we preferred bilateral injections in the TFESI group.

Manchikanti et al. [10] divided 120 patients with CSS into two groups and performed repeated ILESI with a mixture of steroid and local anesthetic in one group and only local anesthetic in the other group. After a 2-year follow-up, they reported significant improvement in pain and functional status in both groups. Although our study differs from this study, as we had a shorter follow-up, used only a combination of local anesthetic and a steroid, and preferred a single ILESI, we achieved, similar to the mentioned study, a significant relief in pain intensity and improvement in the functional status in all follow-up examinations in the ILESI group. In addition, in these patients, we observed improvement in the depression scores and an increase in the walking distance, which had been impaired due to the NC. We believe that these positive results depended on the preference of ILESI with parasagittal technique directed to the site, where the complaints of the patient were more prominent [15].

Lee et al. [4] compared TFESI and ILESI in patients with spinal stenosis and disc herniation. TFESI provided a greater decrease in pain and Roland 5-point scores, and improved NRS scores $(\geq 50 \%$ decrease in the fourth month) in spinal stenosis patients, although there was no statistically significant difference between these two techniques in patients with disc herniation. Smith et al. [5] conducted a retrospective case-control study comprising 19 patients treated with TFESI and 19 patients treated with ILESI. They compared the results of TFESI and ILESI 
in patients with radicular pain and CSS, but they could not observe any significant difference between the groups, although pain scores decreased significantly just after the injection, and in the third and sixth-month follow-up. Unlike the results of these studies, we found that all follow-up parameters showed more improvement in the ILESI group compared to the bilateral TFESI group. This result might depend on there being only axial pain in our patients. Although the short-term efficacy of the TFESI on the radicular pain has been reported, the efficacy on axial pain is still under debate [16].

In contrary to the opinion of many physicians that in lumbar ILESI there is a distribution towards the cranial side and no significant distribution towards the caudal side, Weil et al. [17] suggested that in $76 \%$ of cases, the contrast agent was distributed to more than one vertebral level independent of the needle location. Therefore, compared to TFESI, ILESI may be more beneficial in patients with CSS, as a higher volume of the injectate can be administered, and the injectate is distributed to a broader area. This might also be the reason for the better results we obtained in the ILESI group compared to the TFESI group. In addition, taking into consideration the easier application of ILESI from the point of the methodological view, and life-threatening complications reported for TFESI, we believe ILESI is a better therapeutic alternative in LCSS patients [18].

The DN4 is widely used in the studies published in the literature, which were focused on the evaluation of several treatment methods performed for chronic low back pain associated with NP $[19,20]$. It is a suitable tool with a very good sensitivity $(83 \%)$ and specificity $(90 \%)$ to evaluate the presence of NP in chronic back pain [21]. Therefore, in our study, we preferred the DN4 to evaluate the presence of NP and the efficacy of the injection methods on NP. However, although the DN4 is a good alternative to evaluate NP, there is the possibility of missing $20 \%-30 \%$ of patients. In addition, although the DN4 can assess the presence of NP, it cannot provide any insight considering its severity [22].

Comprehensive epidemiological studies demonstrated that $12 \%-55 \%$ of chronic low back pain patients had also NP components. In our study, we determined NP in $58.2 \%$ of patients with chronic low back pain caused by LCSS with the DN4 before the treatment [23]. This result was consistent with the literature. In addition, in the pretreatment period, NP was observed in $69.7 \%$ and $47.1 \%$ of patients that had undergone TFESI and ILESI, respectively. The better results in all post-treatment follow-up parameters in the ILESI group, compared to the TFESI group, might be related to the higher rate of the neuropathic component in the TFESI group. The possible negative effects of the neuropathic component on therapeutic responses might be a result of the structural changes in the synapses of the spinal conus, interneurons, and glial cells, and central sensitization mechanisms related to the glial activation [24].

In our study, although we did not observe any significant change in the number of NP patients in the ILESI group, there was a significant decrease in the TFESI group in the short-term (3 wk) follow-up. Rados et al. [8] evaluated the efficacy of TFESI and ILESI in chronic radiculopathy, and reported that the NP component decreased significantly during the 6-month follow-up, and there was no statistically significant difference, although the decrease was more prominent in the TFESI group. These different results might depend on the heterogeneous study population (different etiological factors), evaluation of the presence of NP evaluated with the PD-Q, and repeated epidural injections. However, the outstanding effect of TFESI on NP in these studies might be explained with the applicability and efficacy of the injection around the dorsal root ganglion and nerve roots, which play an important role in the pain development mechanisms [25].

Finally, one of the points to be considered is the use of a particulate steroid. Because of the risk of accidental intravascular injection and consequent neurological injuries, non-particulate steroids have been recommended, instead of a particulate steroid, in recent years. Clements et al. [26] designed a cross-sectional survey study composed of 314 physicians, who were members of the Spine Interventional Society, and approximately $41 \%$ reported using particulate steroids for lumbar TFESI, and 74\% for lumbar ILESI, indicating the variability in clinical practice. Chatterjee et al. [27] reported that using methylprednisolone, a particulate steroid, provides significantly longer pain relief than dexamethasone, a non-particulate steroid. So, it can be said that there is great variability in clinical practice regarding steroid type, according to the risk of complications and duration of pain relief.

There are some limitations in our study, such as the short follow-up time, single-center design, and absence of a placebo group, due to ethical reasons. On the other hand, taking into consideration the prospective design and multidimensional evaluation of the treatment results, our study still makes a significant contribution to the literature. Furthermore, it is a remarkable study as it is-as far as we know-the only study comparing the effects of TFESI and ILESI on the neuropathic component of pain in patients with LCSS.

In conclusion, ILESI and TFESI are reliable treatment options in the management of patients with LCSS, and provide pain relief, functional improvement, increased walking distance, and positive effects on a depressive mood. The comparison of these two epidural injection 
techniques shows that ILESI might be preferred in patients with LCSS because of its easier application and greater effectiveness. However, TFESI might be a better option in patients with more prominent NP.

\section{CONFLICT OF INTEREST}

No potential conflict of interest relevant to this article was reported.

\section{FUNDING}

No funding to declare.

\section{ORCID}

Savas Sencan, https://orcid.org/0000-0001-8150-0581

Ipek Saadet Edipoglu, https://orcid.org/0000-0002-3510-5991

Alp Eren Celenlioglu, https://orcid.org/0000-0001-8979-0392

Gunay Yolcu, https://orcid.org/0000-0002-8545-7821

Osman Hakan Gunduz, https://orcid.org/0000-0002-3214-803X

\section{REFERENCES}

1. Kalichman L, Cole R, Kim DH, Li L, Suri P, Guermazi A, et al. Spinal stenosis prevalence and association with symptoms: the Framingham Study. Spine J 2009; 9: 545-50.

2. Hall S, Bartleson JD, Onofrio BM, Baker HL Jr, Okazaki H, O'Duffy JD. Lumbar spinal stenosis. Clinical features, diagnostic procedures, and results of surgical treatment in 68 patients. Ann Intern Med 1985; 103: 271-5.

3. Chad DA. Lumbar spinal stenosis. Neurol Clin 2007; 25: 40718.

4. Lee JH, An JH, Lee SH. Comparison of the effectiveness of interlaminar and bilateral transforaminal epidural steroid injections in treatment of patients with lumbosacral disc herniation and spinal stenosis. Clin J Pain 2009; 25: 206-10.

5. Smith CC, Booker T, Schaufele MK, Weiss P. Interlaminar versus transforaminal epidural steroid injections for the treatment of symptomatic lumbar spinal stenosis. Pain Med 2010; 11: 1511-5.

6. Baron R, Binder A. [How neuropathic is sciatica? The mixed pain concept]. Orthopade 2004; 33: 568-75. German.

7. Atlas SJ, Deyo RA, Patrick DL, Convery K, Keller RB, Singer DE. The Quebec Task Force classification for spinal disorders and the severity, treatment, and outcomes of sciatica and lumbar spinal stenosis. Spine (Phila Pa 1976) 1996; 21: 2885 92.
8. Rados I, Sakic Zdravcevic K, Hrgovic Z. painDETECT questionnaire and lumbar epidural steroid injection for chronic radiculopathy. Eur Neurol 2013; 69: 27-32.

9. Schizas C, Kulik G. Decision-making in lumbar spinal stenosis: a survey on the influence of the morphology of the dural sac. J Bone Joint Surg Br 2012; 94: 98-101.

10. Manchikanti L, Cash KA, McManus CD, Damron KS, Pampati V, Falco FJ. A randomized, double-blind controlled trial of lumbar interlaminar epidural injections in central spinal stenosis: 2-year follow-up. Pain Physician 2015; 18: 79-92.

11. Smith CC, McCormick ZL, Mattie R, MacVicar J, Duszynski $\mathrm{B}$, Stojanovic MP. The effectiveness of lumbar transforaminal injection of steroid for the treatment of radicular pain: a comprehensive review of the published data. Pain Med 2020; 21: 472-87.

12. Farooque M, Salzman MM, Ye Z. Effectiveness of bilateral transforaminal epidural steroid injections in degenerative lumbar spinal stenosis patients with neurogenic claudication: a case series. PM R 2017; 9: 26-31.

13. Davis N, Hourigan P, Clarke A. Transforaminal epidural steroid injection in lumbar spinal stenosis: an observational study with two-year follow-up. Br J Neurosurg 2017; 31: 2058.

14. Scapinelli R. Anatomical and radiologic studies on the lumbosacral meningo-vertebral ligaments of humans. J Spinal Disord 1990; 3: 6-15.

15. Ghai B, Bansal D, Kay JP, Vadaje KS, Wig J. Transforaminal versus parasagittal interlaminar epidural steroid injection in low back pain with radicular pain: a randomized, doubleblind, active-control trial. Pain Physician 2014; 17: 277-90.

16. Manchikanti L, Abdi S, Atluri S, Benyamin RM, Boswell MV, Buenaventura RM, et al. An update of comprehensive evidence-based guidelines for interventional techniques in chronic spinal pain. Part II: guidance and recommendations. Pain Physician 2013; 16(2 Suppl): S49-283.

17. Weil L, Frauwirth NH, Amirdelfan K, Grant D, Rosenberg JA. Fluoroscopic analysis of lumbar epidural contrast spread after lumbar interlaminar injection. Arch Phys Med Rehabil 2008; 89: 413-6.

18. Tahmasbi Sohi M, Sullivan WJ, Anderson DJM. Transient hypokalemic quadriplegia after a lumbar transforaminal epidural dexamethasone injection: a case report. PM R 2018; 10: 544-7.

19. Sarı S, Aydın ON, Güleser G, Kurt İ, Turan A. Effect of transforaminal anterior epidural steroid injection on neuropathic pain, quality of sleep and life. Agri 2015; 27: 83-8.

20. Batistaki C, Angelopoulou A, Smyrnioti ME, Kitsou MC, Kostopanagiotou G. Electromyographic findings after epidural steroid injections in patients with radicular low back pain: a prospective open-label study. Anesth Pain Med 2017; 7: e62556.

21. Kim HJ, Park JH, Bouhassira D, Shin JH, Chang BS, Lee CK, 
et al. Validation of the Korean version of the DN4 diagnostic questionnaire for neuropathic pain in patients with lumbar or lumbar-radicular pain. Yonsei Med J 2016; 57: 449-54.

22. Gudala K, Ghai B, Bansal D. Usefulness of four commonly used neuropathic pain screening questionnaires in patients with chronic low back pain: a cross-sectional study. Korean J Pain 2017; 30: 51-8.

23. Andrasinova T, Kalikova E, Kopacik R, Srotova I, Vlckova E, Dusek L, et al. Evaluation of the neuropathic component of chronic low back pain. Clin J Pain 2019; 35: 7-17.

24. Kim JH, Hong JT, Lee CS, Kim KS, Suk KS, Kim JH, et al. Prevalence of neuropathic pain and patient-reported outcomes in Korean adults with chronic low back pain resulting from neuropathic low back pain. Asian Spine J 2017; 11: 917-27.
25. Bogduk N. Practice guidelines for spinal diagnostic and treatment procedures. 2nd ed. San Francisco (CA), International Spine Intervention Society. 2013. pp 459-538.

26. Clements N, Vydra D, Cushman DM, Nagpal A, Julia J, Zheng $\mathrm{P}$, et al. Trends in steroid agent and diluent choices for epidural steroid injections: a survey of Spine Intervention Society physicians. Reg Anesth Pain Med 2019. doi: 10.1136/ rapm-2018-100366.

27. Chatterjee N, Roy C, Das S, Al Ajmi W, Al Sharji NS, Al Mandhari A. Comparative efficacy of methylprednisolone acetate and dexamethasone disodium phosphate in lumbosacral transforaminal epidural steroid injections. Turk J Anaesthesiol Reanim 2019; 47: 414-9. 